\title{
Epilepsy Identification using EEG signal monitoring
}

\author{
Ganesh Birajadar $^{\mathrm{a}}$ and Dr. Channappa Bhyri ${ }^{\mathrm{b}}$

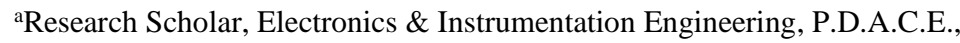 \\ Kalaburagi, VTU, India.

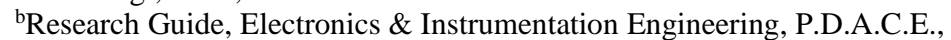 \\ Kalaburagi, VTU, India.
}

Article History: Received: 11 January 2021; Accepted: 27 February 2021; Published online: 5 April 2021

\begin{abstract}
Electroencephalogram (EEG) is nothing but measuring electric activity of brain. EEG is non-stationary signal. EEG characterizes human behavior. There are many brain abnormalities that can be identified and treated using EEG behavior analysis. As per researchers study Epilepsy is commonly happening disorder that is getting spread over the time. It is nothing but sudden stroke in brain where patient suffers from unusual activities seizures. Sometimes symptoms are such severe that ignorance leads to death. So it is important to identify its earlier symptoms and treat it in time so as to avoid risk. EEG signals are used for getting features in time as well as frequency domain. These features are further analyzed and classified to identify EEG abnormality.
\end{abstract}

Keywords: EEG, Epilepsy, non-stationary, Abnormality, features.

\section{Introduction}

EEG signal plays important role in identifying various brain disorders. Epilepsy is considered as a most usually occurring brain abnormality that, according to W.H.O. evaluation, touches nearly 0.06 billion folks all over the biosphere. Roughly one amongst hundred persons gets suffered from seizure during his/her lifespan. This disorder is described by the repeated and abrupt occurrence having epileptic seizures which may result in to unsafe and conceivably deadly conditions. EEG shows electrical movement of the brain which consists of transitory and unanticipated electrical disruption of the brain and disproportionate neuronal release that ends in epileptic stroke. Subsequently, the EEG signal can be treated as most exploited signal in medical investigations which gives an idea of the brain state and also helps clinicians' for early detection of epileptic strokes which plays vital role in early diagnosis and precautionary measures.

Whenever patient consults physician, patient's EEG data is generally acquired for a few days which is a tiresome and inefficient process. The revealing of abnormality in brain just by observing reports is time consuming and also difficult for physician to diagnose on a large scale. In spite of that, it also needs a professional to examine the complete span of the EEG records, so as to identify abnormal activity in EEG. A trustworthy involuntary classification and recognition scheme would guarantee an objective with enabling treatment and suggestively advance an epilepsy diagnosis in addition to that long-term monitoring and treatment of patients. Consequently, there arises a solid demand in the improvement of similar programmed systems, due to both massive quantities and augmented habit of long-standing EEG records for appropriate assessment and cure of neurological illnesses, comprising epilepsy. It can narrow down the possibility of the professional misunderstanding the statistics and failure to make correct decision.

In recent era, numerous automated brain wave sorting and seizure recognition systems have developed. These use various algorithms for finding disorders. One of the researchers named Gotman offered a high-tech system for perceiving different seizure types. Meantime $\mathrm{Qu}$ and Gotman projected their findings by using k nearestneighbor classification algorithm on $\mathrm{t} \& \mathrm{f}$ domain extracted features of EEG. It is useful in detecting seizure onset of epilepcy. Gigola and his coworkers used an evolutionary method which uses gathered energy. They used wavelet decomposition for predicting onset of epileptogenic seizure from intracranial EEG records. Adeli along with Guler proved time series potential in nonlinear analysis in early detection.

Many researches proposed their concepts which are based on ANN grounded detection schemes for identifying epilepsy. Weng along with Khorasani used Gotman discussed features like average of brain wave magnitude, averaged duration of EEG, coefficient of variation, power spectral average and frequency dominance. They treated these features as source of inputs for adaptive NN. Pradhan with his researchers focused on raw EEG which is used as input to learning vector quantization system. Nigam came with proposing innovative NN model named Large Memory Storage and Retrieval type of network and 2 spatial domain EEG characteristics. These characteristics are spike rhythmicity and comparative amplitude that acted as inputs to the seizure detection algorithm. The procedure projected by Kiymik uses BPNN using AR coefficients and spectral density estimate 
treated as responses for programmed recognition of seizures. Ghosh Dastidar conferred new methodology of classification which is relevant with wavelet decomposition which contained and LMBPNN with basis function (radial). Srinivasan offered a methodology which took help of imprecise entropy as response for ANN classifier. Subasi focused on wavelet based analyzing and experts mixture along with ANN, to categorize normal EEG from epileptic EEGs..

\subsection{EEG signal}

To acquire electric activity of brain, EEG researchers use different invasive or noninvasive methods. They do not need to insert electrodes in skulls during noninvasive method so it is easier. Appreciatively, electrodes can be arranged on scalp with reference electrodes and electric activity of brain can be recorded easily. German neurologist Hans Berger applied this procedure to humans in 1920s. EEG is hence proved to be least expensive, non-invasive, and entirely passive recording method.

As compared to other imaging techniques like FMRI, EEG proved more beneficial and gives more clean behavior interpretations. EEG represents outstanding time resolution, which means, it is able to take numerous of electrical activity snapshots using different electrodes in fraction of a second. This solidifies brain signal as perfect tools for learning the specific time base of mental and intellectual behavioral treating.

In distinction to extra physiological records like EDA/GSR that frequently necessitate merely very few of electrodes. EEG records are obtained using electrode arrangements, including numerous sensor figures fluctuating from ten to more than five hundred electrodes, dependent on the latitude of the experiment. For quicker solicitation, elastic caps of pre mounted electrode locations, meshes, or rigid grids, are used safeguarding that values obtained are from matching scalp locations through respondents.

\subsubsection{EEG subbands}

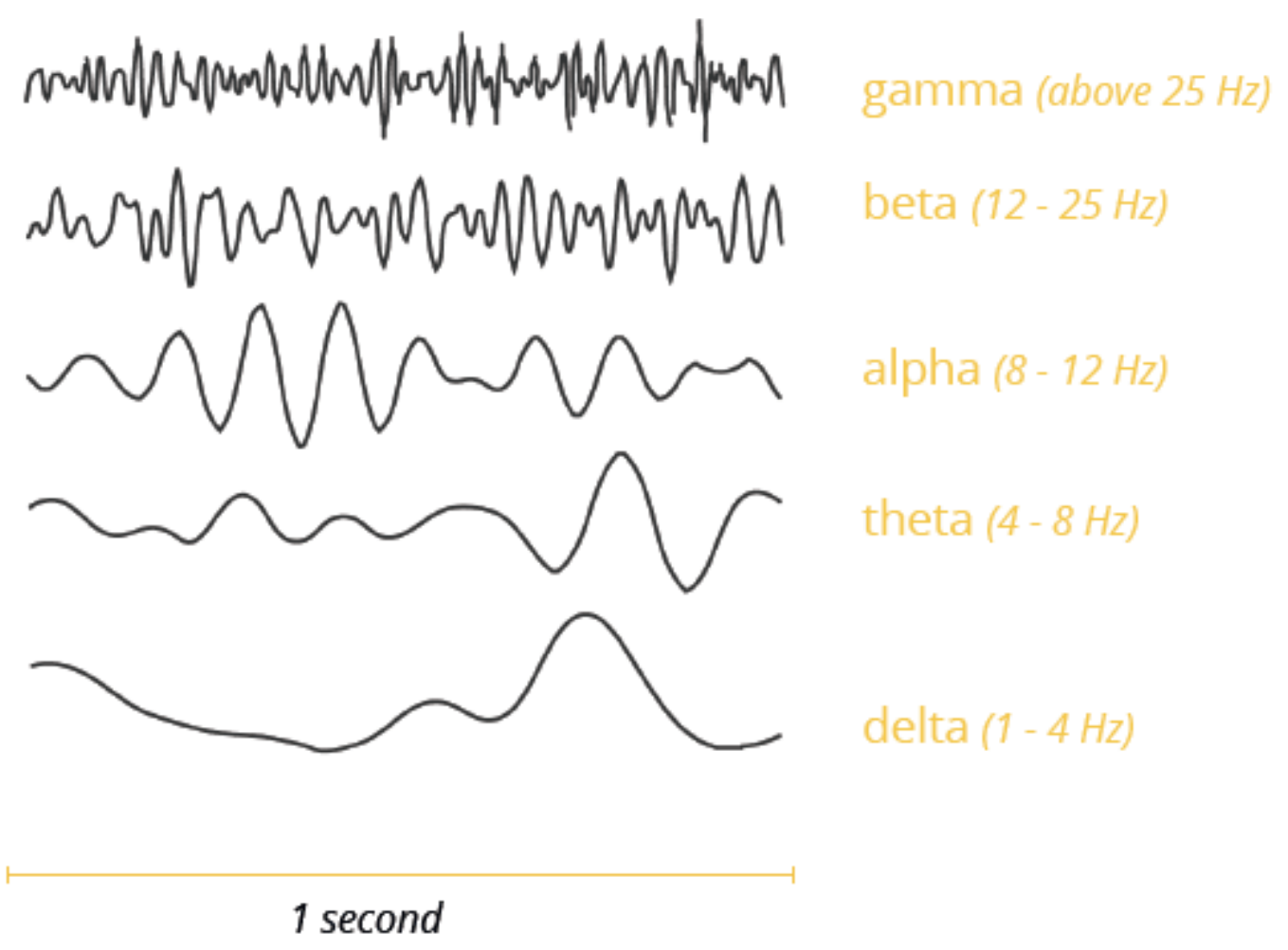

Figure 1. EEG sub bands

\subsection{EEG signal acquisition : The 1020 Electrode System}

The 1020 system consists of placement of electrodes that covers brain in such a way that The perpendicular link joining front (nasion) with back (inion) plus parallel link joining right with left pre-auricular sockets are distributed into 10 identical sectors. Likewise, equator is distributed into ten per cent and twenty per cent lots.

Important points in the 10-20 scheme are:

- $\quad$ Nasion $(\mathrm{Nz})$

- Inion (Iz)

- Rightward and Leftward preauricular points 


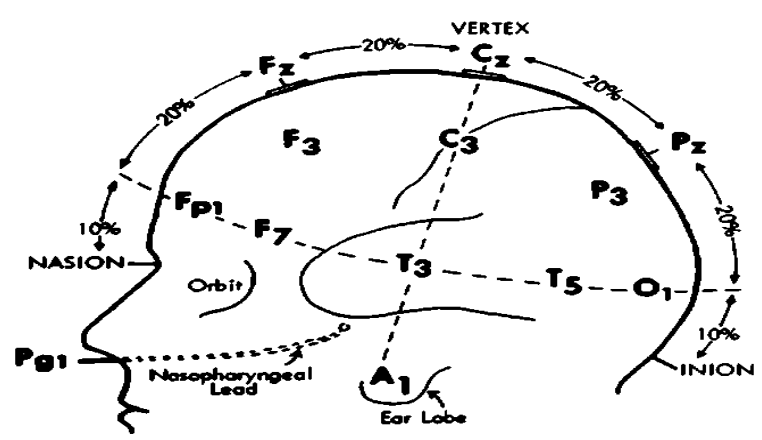

LEFT SIOE OF HEAO

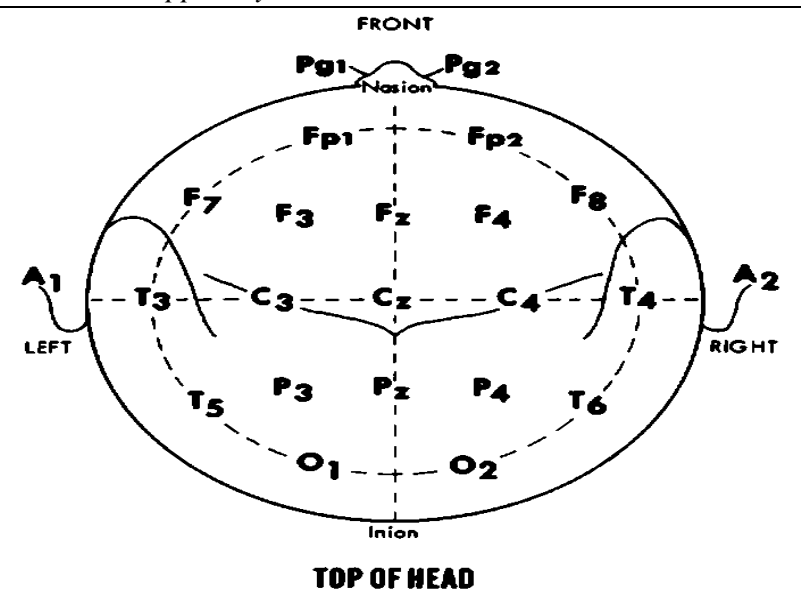

TOP OF HEAD

\subsection{Artifacts}

Figure 2. 1020 Electrode System

Before researcher hurdle into EEG acquisition and its analysis, one thing should be considered that whatever data is acquired should be purely clean from unwanted disturbances so as to get accurate results. Always it should be ensured that collected data must be free from artifacts as much as possible, which concludes that acquired data returns brain electric values only. Theoretically as it sees very simple but practically it is very much tedious and carefully treated task. The various artifacts are listed below.

1.3.1. Physiological Artifacts
(1) Muscle Activity
(2) Eye Movements
(3) Blinks

\subsubsection{External Sources of Artifacts}

(1) Electrode Movement

(2) Line Noise

(3) Swinging

As scalp electrodes can collect electrical movement starting at different places in atmosphere, its advised to circumvent, diminish or at least govern such categories of artifacts as finest as possible.

\section{Methodology}

As it is discussed earlier EEG signal is acquired using 1020 electrode system. Acquired signal is always contaminated with unwanted signals known as artifacts. Then acquired signal is filtered using various filters depend on presence of artifacts. Filtered EEG signal is used for extracting features from it. As one domain cannot give the desired information, signal is analyzed in time as well as frequency domain. For example features extracted are mean, variance, kurtosis, skewness, bandpower, entropy, wavelet band energy, wavelet coefficients, etc. Acquired features are further studied and selected for further classification. Different classifiers like ANN, SVM, CNN can be used for getting better accuracy.

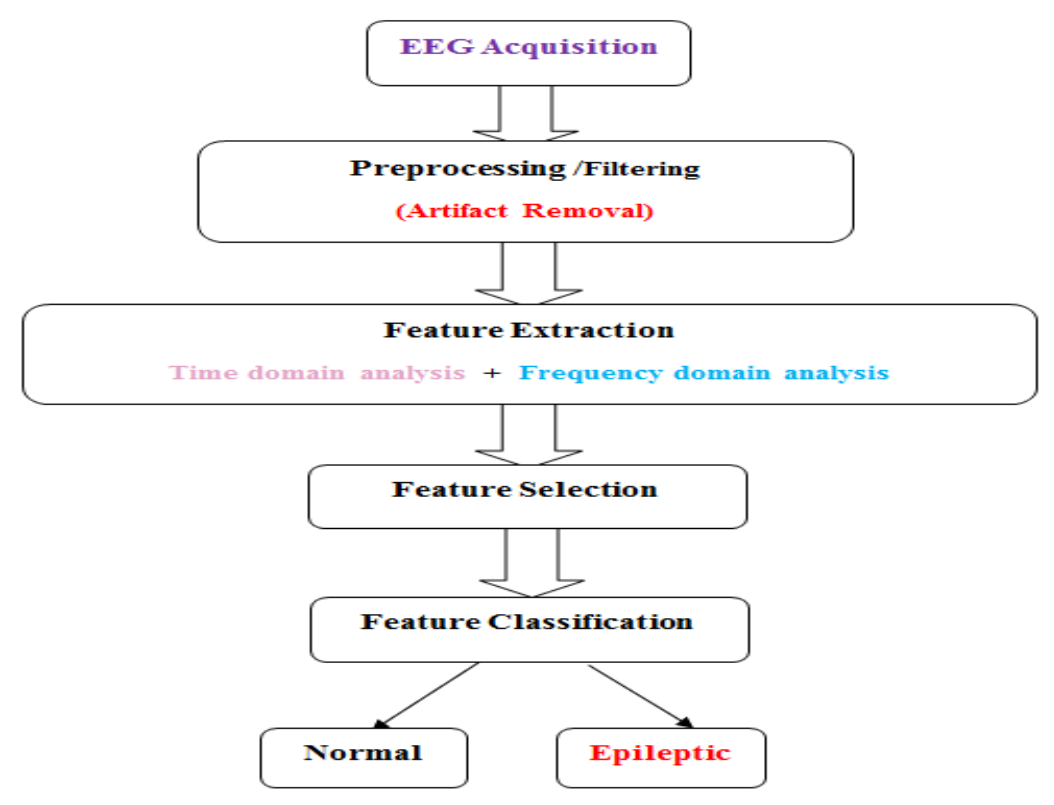


Figure 3. Methodology

\section{Features Extraction}

\section{- Kurtosis}

$$
\text { Kurtosis }=\frac{M_{4}[x(n)]}{M_{2}^{2}[x(n)]}
$$

Where $M_{i}[x(n)]$ is $\mathrm{i}^{\text {th }}$ central moment of $\mathrm{x}(\mathrm{n})$

$$
M_{i}[x(n)]=E\left[(x(n)-\mu)^{i}\right]
$$

For normal distribution $\mathrm{K}=3$

Excess or Normalized kurtosis is given as

$$
E x_{\text {Kurtosis }}=\frac{M_{4}[x(n)]}{M_{2}^{2}[x(n)]}-3
$$

\section{- Skewness}

$$
\text { Skewness }=\frac{E\left[(x(n)-\mu)^{3}\right]}{\sigma^{3}}
$$

\section{- Wavelet Energy}

For each level $\mathrm{i}=1, \ldots . l$ (No of decomposition levels)

$$
\begin{gathered}
E_{D_{i}}=\sum_{j=1}^{N}\left|D_{i j}\right|^{2}, \quad i=1,2,3, \ldots, l \\
E_{A_{i}}=\sum_{j=1}^{N}\left|A_{i j}\right|^{2}, \quad i=l
\end{gathered}
$$

Total energy is given as

$$
E_{\text {Total }}=\left(\sum_{i=1}^{l} E_{D_{i}}+E_{A_{i}}\right)
$$

Normalised energy is given as

$$
E_{\tau}=\frac{E_{j}}{E_{\text {Total }}}
$$

\section{Results}

Figure 4 shows normal and epileptic EEG signals. From this one can see changes occurred during seizure activity.
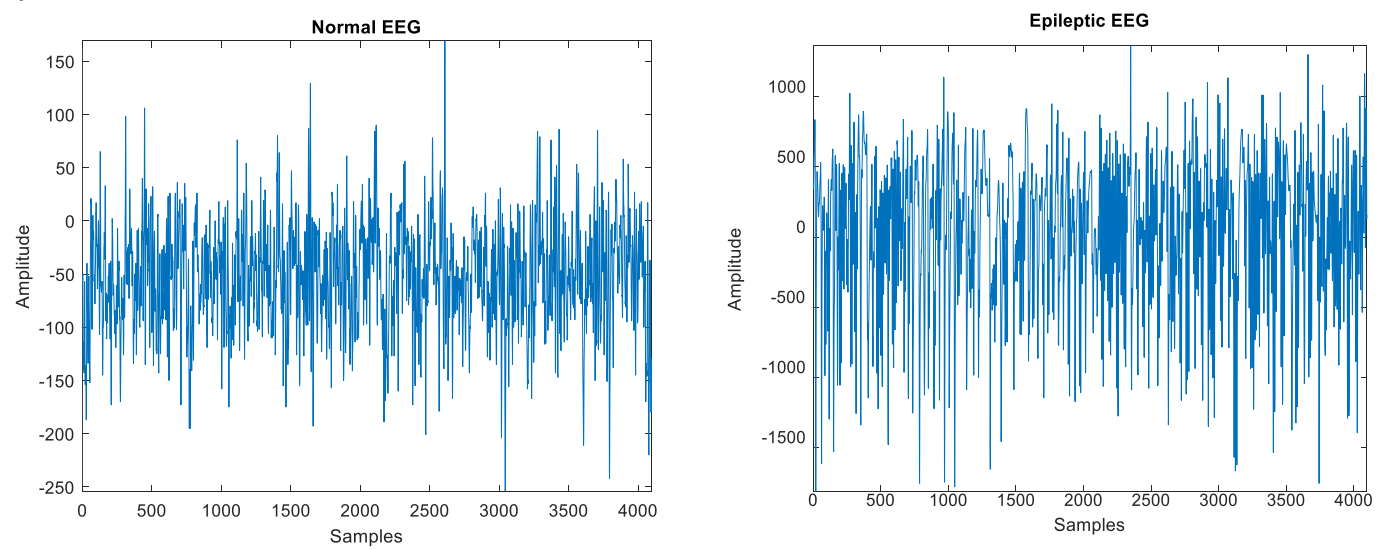
Table 1. Comparison of extracted feature values

\begin{tabular}{llc}
\hline EEG & Feature & Value \\
\hline \multirow{2}{*}{ Normal } & Kurtosis & 3.6256 \\
\cline { 2 - 3 } & Skewness & -0.0927 \\
\hline \multirow{2}{*}{ Epileptic } & Kurtosis & 3.2700 \\
\cline { 2 - 3 } & Skewness & -0.6740 \\
\hline
\end{tabular}

Classifier performance is measured for accuracy, sensitivity, specificity, precision

$$
\begin{gathered}
\text { Accuracy }=\frac{\text { Total no of correctly classified instances }}{\text { Total noof instances }} * 100 \\
\text { Sensitivity }=\frac{\text { True Positive }}{\text { True Positive }+ \text { True Negative }} * 100 \\
\text { Specificity }=\frac{\text { True Negative }}{\text { False Positive+True Negative }} * 100 \\
\text { Precision }=\frac{\text { True Positive }}{\text { True Positive }+ \text { False Positive }} * 100
\end{gathered}
$$

\section{Conclusion}

EEG signal is used for detecting brain disorders. For EEG signals of Normal and Epileptic patients time and frequency domain features are calculated. Wavelet sub band coding is used with decomposition level 4 using Db2 mother wavelet. The sub band energies are calculated. Also mean , variance Skewness, Kurtosis values are calculated. These features can be used to classify signals. Classifiers like Convolutional Neural Network and Support vector Machine are used and compared their performance for accuracy and precision. It can be concluded that EEG plays its role as a detection tool to detect the disease in the early stage, rehabilitation, classification or as an assistive tool for the patient according to the needs of the diseases.

\section{References}

Sachin M. Elgandelwar, Vinayak K. Bairagi, "Role of EEG for diagnosis of Alzheimer Disease", International Journal of Innovative Technology and Exploring Engineering (IJITEE) ISSN: 2278-3075, Volume-8 Issue-10, August 2019.

Ganesh Birajadar, Channappa Bhyri , "Comparative survey on EEG analyss for detecting brain disorders", International journal of Future Generation Communication and Networking, Vol 13 No. 3s (2020) pp. 12531257.

Ganesh Birajadar, Channappa Bhyri " "Comparative Analysis on Brain Disorder detection using EEG”, International Journal of Innovations in Scientific \& Engineering Research, Vol 6, Issue 6, June 2019.

S. Vani, G. R. Suresh, “EEg Signal Analysis for Automated Epilepsy Seizure Detection Using Wavelet Transform and Artificial Neural Network". Journal of Medical Imaging and Health Informatics, Vol 9, 1301 1306, 2019.

Shitanshu Kusmakar, Chandan K. Karmakar, "Automated Detection of Convulsive Seizures Usinga Single Wrist-Worn Accelerometer Device", EEE TRANSACTIONS ON BIOMEDICAL ENGINEERING, 2019

P. B. Mane and A. O. Mulani, "High throughput and area efficient FPGA implementation of AES algorithm", International Journal of Engineering and Advanced Technology, Vol. 8, Issue 4, April 2019

Ganesh Shinde and Altaf Mulani, "A Robust Digital Image Watermarking using DWT-PCA", International Journal of Innovations in Engineering Research and Technology (IJIERT), Vol. 6 Issue 4 April 2019.

Oleksii Shandra and Alexander R. Winemiller, Repetitive Diffuse Mild Traumatic Brain Injury Causes an Atypical Astrocyte Response and Spontaneous Recurrent SeizuresThe journal of Neuroscience, 24 April 2018.

Muhammad Sohaib J. Solaija, Sajid Saleem, "Dynamic Mode Decomposition Based Epileptic Seizure Detection from Scalp EEG”, IEEE Acess, 2018.

Fera Putrì Ayu Lestari ; Evi Septiana Pane, "EEG seizure detection and prediction algorithms: a survey", 2018 International Conference on Information and Communications Technology (ICOIACT) 2018.

Annegers JF, Coan SP (2000) The risks of epilepsy after traumatic brain injury. Seizure 9:453-457

S. S. Swami and A. O. Mulani, "An efficient FPGA implementation of Discrete Wavelet Transform for image compression", International Conference on Energy, Communication, Data Analytics and Soft Computing (ICECDS), 2017

Mulani Altaf O. and P. B. Mane, "Watermarking and Cryptography based Image Authentication on reconfigurable platform", Bulletin of Electrical Engineering and Informatics, June 2017. 
Mulani Altaf O. and P. B. Mane, "An Efficient implementation of DWT for image compression on reconfigurable platform", International Journal of Control Theory and Applications, Jan. 2017.

Bruno Albert, Jingjing Zhang, "Automatic EEG Processing for the Early Diagnosis of Traumatic Brain Injury," 2016

Prabhapreet Kaur, Anurag Sharma, "Epilepsy Seizure Detection Using Wavelet Support Vector Machine Classifier", International Journal of Bio science \& Bio technology, Vol. 8 No. 2, 2016. 